\title{
Discharge amplified photo-emission from ultra-thin films applied to tuning work function of transparent electrodes in organic opto- electronic devices
}

\author{
A.R. Gentle ${ }^{1}$, G.B. Smith ${ }^{{ }^{*}}$ and S.E. Watkins ${ }^{2}$ \\ ${ }^{1}$ Physics and Advanced Materials, University of Technology, Sydney, \\ PO Box 123, Broadway, NSW, 2007 Australia \\ ${ }^{2}$ CSIRO Materials Science and Engineering, Clayton South, Victoria 3169, Australia \\ Email: g.smith@uts.edu.au
}

\begin{abstract}
A novel photoemission technique utilizing localized discharge amplification of photo-yield is reported. It enables fast, accurate measurement of work function and ionization potential for ultra-thin buffer layers vacuum deposited onto single and multilayer transparent conducting electrodes for organic solar cells and OLED's. Work function in most traditional transparent electrodes has to be raised to maximise charge transfer while high transmittance and high conductance must be retained. Results are presented for a range of metal oxide buffers, which achieve this goal. This compact photo-yield spectroscopy tool with its fast turn-around has been a valuable development aid since ionization potential can vary significantly as deposition conditions change slightly, and as ultra-thin films grow. It has also been useful in tracking the impact of different post deposition cleaning treatments along with some storage and transport protocols, which can adversely reduce ionization potential and hence subsequent device performance.
\end{abstract}

Keywords: photo-emission, ionization potential, discharge amplification, transparent electrodes, OPV/OLED

*Corresponding Author. Tel.: +61 295142224

E-mail address : g.smith@uts.edu.au (G.B. Smith) 


\section{Introduction}

Techniques for measurement of work function (WF) and ionization potential (IP) continue to be of interest and have evolved recently to deal with nanostructures using vibrating AFM tips and STM probes [1]. However photoemission (PE) remains the most widely used technique. The method presented represents an advance in photo-yield spectroscopy (PYS) utilising discharge amplification of photo-yield induced by a low energy UV source. Its operational principles parallel those of the gaseous secondary electron detector (GSED) as used in environmental scanning electron microscopes. These detectors enable quality imaging in various atmospheres.

The work function of conducting contacts are needed for traditional junction devices, in particular the differences between WF value of the electrode and that of the semiconductors used. The latter's replacement with organic [2] and organometallic conductors, and their use in various optoelectronic systems has driven new demands on both work function or ionization potential values and their measurement. Depending on just how contacts are made, and whether they are anode or cathode, work function or ionization potential may be involved. IP is relevant for many oxide electrode buffer layers as they are semiconductors not conductors. That is in their ground state they are insulators and their filled levels do not traverse the Fermi level so that electron or hole conduction occurs via thermal activation. Several PE examples given in this paper are for such oxides. They are very thin and used to facilitate charge transfer between the transparent conducting electrode and the active organic system. Our PYS device can measure both WF for conductors and IP for semiconductors. For buffer oxides confusion has arisen in the literature with some authors using the term "IP", but many use "WF". Traditionally IP has referred to transitions from the top of the valence band in semiconductors to the vacuum. In sub-stoichiometric transition metal oxides however the highest occupied states arise from defect states that form within the pure oxide band gap. Thus traditional photo-excitation from the valence band and from gap states are both present. As long as the oxide is a semiconductor two classes of ionization potential thus exist, the traditional one and that resulting from the presence of gap states. It is important to know when IP and when WF are given by PE spectra. Given the misunderstandings that exist we introduce here new nomenclature and acronyms : IPV and IPD to define respectively, ionization potential from valence states, and ionization potential due to defect or gap states, with IP used when a combination occurs. Both contribute to our data.

For many organic opto-electronic devices maintenance of high ionization potential is essential for the anode. For organic solar cells (OPV) and OLEDS at least one contact needs to combine three or more attributes. High conductivity, high transmittance over a bandwidth dictated by the active material, and a narrow range of IPD are the prime concerns, but other features such as flexibility and stability against changes in IPD induced by atmospheric conditions, or cleaning either chemically or with discharge ions, may also be needed. Highly flexible electrodes are of interest for OPV and they also enable roll-to-roll production of complete OPV systems. Good flexibility usually demands much thinner layers than are used to achieve the desired conductance in traditional transparent conducting oxide (TCO) films such as indium tin oxide (ITO) and aluminium doped zinc oxide (AZO). Multilayers involving anti-reflected very thin silver are one approach to achieving desired electrical and optical responses in flexible sub-100 nm total thicknesses. While high ionization potential oxides can be used to achieve anti-reflectance of the silver their conductivity is not as high as one would like. An alternative is to anti-reflect with a TCO material and buffer this with a high IP layer only a few nm 
thick. An example emission spectrum and IP result on such a four-layer electrode using our PYS technique are presented later.

The aim in the multilayer is thus to optimize surface ionization potential while retaining high conductivity and high transmittance. A sufficiently thin transparent outer buffer layer can do this even if its material is only a moderate conductor. Buffer layer materials of interest include $\mathrm{MoOx}$, $\mathrm{ZnO}, \mathrm{WO}_{3}, \mathrm{TiO}_{2}$ and $\mathrm{V}_{2} \mathrm{O}_{5}$ [3]. It is thus important to know the IP of such thin, vacuum deposited layers, which should remain around $5 \mathrm{~nm}$ or less in thickness. Very thin films of these oxides when sputtered or evaporated may have different structures, stoichiometry and defect content to the outer layers of their thicker counterparts. Such changes modify work function, and also carrier densities and mobilities. Any surface modifications must also not induce any other additional barriers for charge transfer as these can degrade I-V characteristics and hence performance.

Traditional approaches to photoemission such as Ultraviolet Photoelectron Spectroscopy (UPS) require relatively thick films of sufficient conductivity to minimise probing of the substrate and charging. An attraction of the PYS technique is that it is performed in an atmosphere in the 1-5 mTorr range so charging of the sample becomes significantly less of a problem. The sample set up described later also helps eliminate charging as an issue. Thus the need for expensive UHV equipment is avoided. It utilises a scanned low energy mono-chromatic UV source at 4-7eV, [4, 5] rather than a high energy $22.1 \mathrm{eV}$ source as used in UPS. As a verification of the lack of charging effects photoemission spectra on standard insulators, semiconductors and strong conductors were all found to be as expected and in agreement with accepted values. It exploits the same processes which helps make the environmental scanning electron microscope viable as a high resolution imaging system.

\section{Material and methods}

This approach to PYS utilises the gas cascade, which occurs when an electron is emitted from the sample surface, allowing us to measure the positive ion current as the nearest path to ground is through the pico-ammeter [5,6]. The layout must be carefully designed to ensure the discharge occurs through to the desired electrode rather than to other grounded areas of the chamber. To assist in this Teflon sheeting is used for electrical isolation to cover any other grounded surfaces near the probe and sample stage.

A wide variety of materials are of interest for use in flexible transparent conducting electrodes including mixed oxides and nano-composites, while different deposition conditions and surface treatments are also used in the search of optimized electrodes. This means that in development of transparent conducting electrodes there is a need for rapid and accurate analysis. This compact instrument enables many samples to be measured and analysed in a short period. 


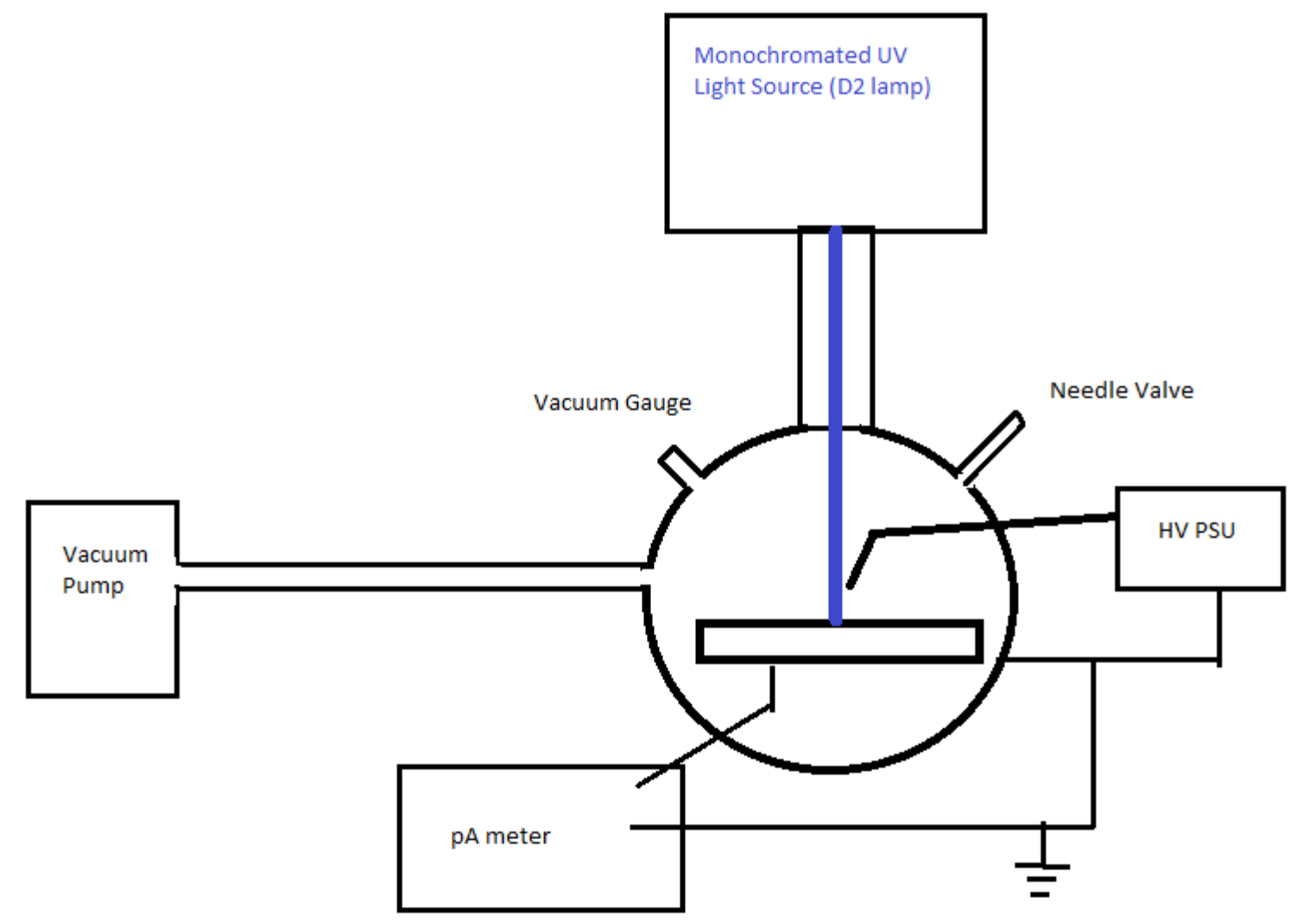

Figure 1: Schematic of the PYS setup

Our system, as depicted in figure 1, consists of a StelaNet SL3 Deuterium Lampand JobinYvon HR 250 monochromator with stepper motor to control the wavelength of the incident light. The output of the monochromator is focused downwards using a parabolic mirror tilted at $45^{\circ}$ after the output slit. The vacuum chamber has a quartz window to allow exposure of the test sample to the UV light. Our overall design aims to optimize signal amplification, rather than it simply being a side effect of measuring in a gaseous environment. The positive electrode consists of a copper wire directed towards the sample stage at a spacing of 3-5 mm with a high voltage feed through, connected to a variable high voltage power supply set in the range of $500-600 \mathrm{~V}$. The sample stage is a sheet of stainless steel embedded in a polycarbonate frame such that it is electrically isolated from the rest of the chamber. This feature plus the sharp probe, its placement, the gas flow, the voltages used and insulation of chamber walls, ensure that the ionization of the gas is controlled so that fields and hence ionization is localized and occupies a small fraction of the view from any point on the wall. Breakdown is prevented and stray signal resulting from secondary emission off the walls is also prevented by the higher than normal pressures and weak or absent field strength near the walls. No visible discharge is apparent but the voltage drop developed at the high gas pressures and gas flow regime used is enough to cause some ionization and cascading, hence amplification, but not enough to induce breakdown. If pressure was reduced by a moderate amount, applied voltage raised too much above the set range, or gas flow cut-off, the instrument would no longer function as intended. Thus 
the overall spatial design, plus operational settings and their control, are each important. Results on a variety of oxide, semiconductor and metal layers including some standards, confirm that ion impact damage is as expected, not a problem.

The chamber is evacuated using a rotary pump. An air inlet valve positioned such that the gas flow flushes past the electrode and stage, is used to set the desired pressure (1-5 mTorr) to maximise amplification [7]. Currently our set-up is used for testing samples in an air atmosphere, though testing in other atmospheres is a trivial matter of attaching the desired carrier gas to the inlet valve. We have continued to use air as it gives a good indication of surface contaminants that may get onto samples when processed into devices. It is essential to monitor the pressure of the chamber and ensure that it is stable, as variations in pressure will affect the gain of the gas-cascade amplification.

Upon illumination with the UV light electrons are emitted from the sample surface. As each of these electrons, accelerated by the local applied electric field, strike molecules in the atmosphere the gas molecules are ionised thereby liberating further electrons to continue the cascade process towards the positive electrode. As noted above this method is much the same as that used to produce the secondary electron signal in environmental scanning electron microscopes [6]. The amplified photoemission current can then be measured by grounding the sample stage through a precision ammeter, in our case a Keithley 617 Electrometer. Measuring the grounded stage rather than the typical method of measuring the current on the high voltage side was done for two reason, firstly the protection of the electrometer from high voltage input and secondly to further eliminate signal from any stray UV light not incident on the sample surface.

As many of our samples are produced on insulating substrates we have found that utilising a strip of copper tape from the rear surface that contacts the stainless steel stage to the front side of the sample is a simple method to eliminate substrate related charging issues. In order to optimise the measurement conditions for each sample type it is convenient to set the monochromator to $200 \mathrm{~nm}$ which is where the peak signal of our samples is acquired. As with materials that have a very high PE yield it is often best initially to vary the pressure or applied voltage so as to ensure there is no breakdown of the carrier gas.

Scanning from $310 \mathrm{~nm}$ to $170 \mathrm{~nm}$ allows the zero signal offset current $\left(I_{\text {offset }}\right)$ to be measured before and after the sample measurement as most of the materials of interest for electrodes have high work function hence they have no photoemission above $310 \mathrm{~nm}$, while our light source has no intensity below $185 \mathrm{~nm}$.

A Hamamatsu 955 photomultiplier was utilized to measure the relative spectral output, and the slit width of the mono-chromatic Deuterium lamp set-up. The wavelength calibration and slit width was determined using the D2 $656.1 \mathrm{~nm}$ emission line. A slit width giving a FWHM of $2 \mathrm{~nm}$ was chosen to give a good balance between signal strength and spectral response. To normalize the data the measured photomultiplier signal was divided by the response curve of the PMT giving the Calibration factor $C F(\lambda)$ for the combined output of the light source and monochromator.

The sample is scanned at a $1 \mathrm{~nm}$ step size from $310-170 \mathrm{~nm}$. The recorded data $I(\lambda)_{\text {Measured }}$ is then corrected for the incident light intensity by:

$\mathrm{I}(\lambda)=\left[I(\lambda)_{\text {Measured }}-\mathrm{I}_{\mathrm{offset}}\right] / \mathrm{CF}(\lambda)$

Photoemission yield $Y(\lambda)$ is traditionally analysed by taking the square root of PE intensity, for metals, and the cube root for semiconductors $[5,8]$. 
Thus

$Y(\lambda)=I(\lambda)^{1 / n}$

The value of $\mathrm{n}=2$ for metals, and $\mathrm{n}=3$ for oxides and semiconductors. The intercept of the linearized yield and the background with respect to the incident photon energy is interpreted as the ionisation potential (IP) for oxides and semiconductors, or work function (WF) for metals.

\section{Results and Discussion}

We have characterised various materials using this technique including some comparisons with data collected using a RKI Industries AC-2 Photo-Electron Spectrometer in Air PESA system [9, 10].The PESA system requires a much higher anode voltage than our technique. A set of example spectra using our PYS method is shown in figure 2 for reactively sputtered molybdenum oxide of various sub-stoichiometries, as produced by varying the deposition current while maintaining a fixed Argon pressure of 1.5 mTorr and varied oxygen gas ratio as seen in the figure. From left to right oxygen content in the films increases which lowers oxygen vacancy defect levels. Oxygen vacancies create defect states which when ionized lead to semiconductor properties. Thus less oxygen tends to raise conductivity while lowering ionization potential as seen in figure 2 .

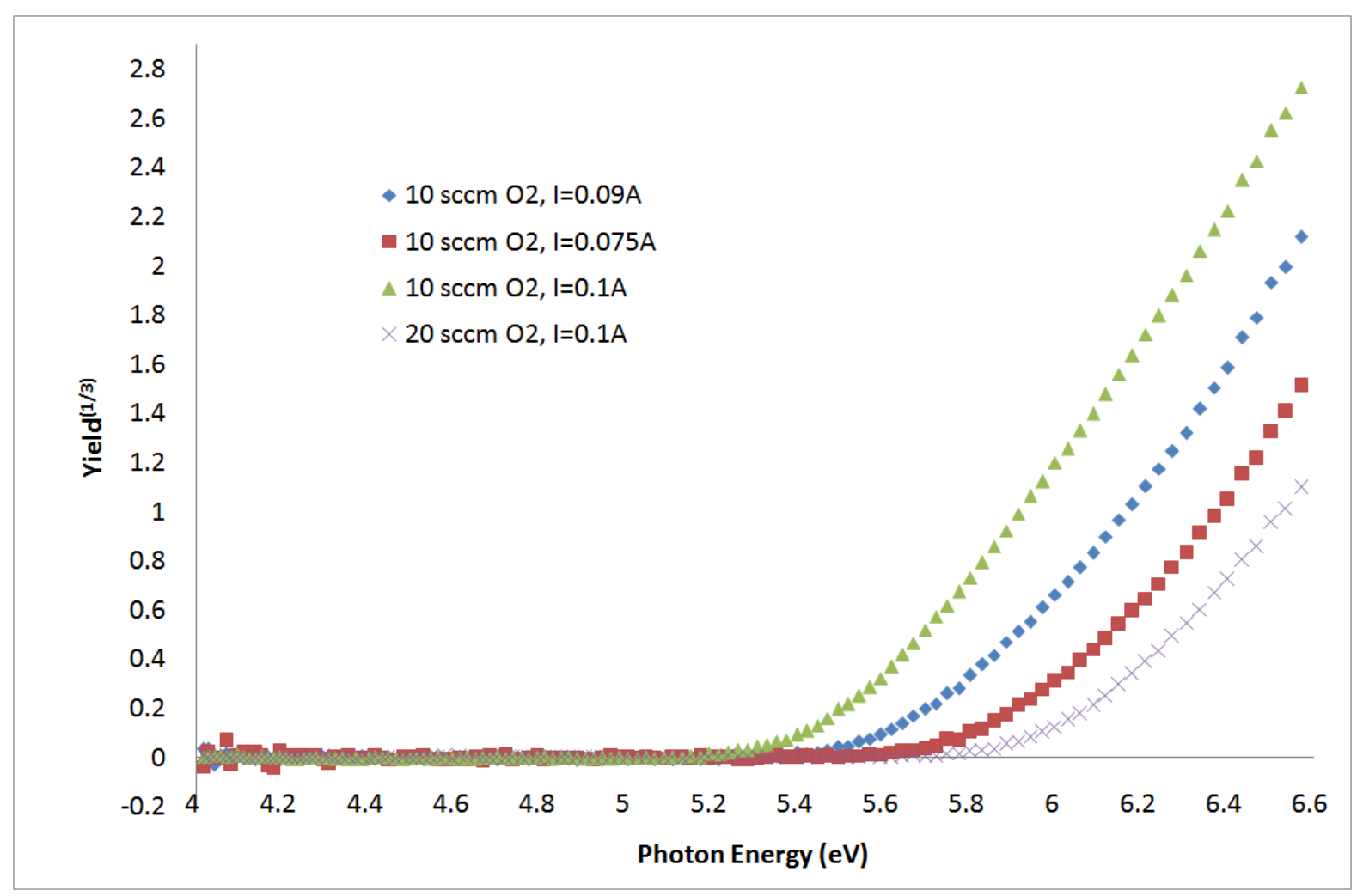

Figure 2: PYS spectra for various 30nm thick MoOx sub-oxides on glass, deposition conditions included in the legend.

In comparisons of IP found with our instrument and by the industry standard PESA AC-2 instrument, we have found good agreement. An example comparison of results from both PESA and PYS measurements are shown in figure 3 . These are for a $5 \mathrm{~nm} \mathrm{MoOx} \mathrm{sample} \mathrm{on} \mathrm{a} \mathrm{glass} \mathrm{substrate,} \mathrm{in}$ which the samples were sputter deposited at the same time. The PESA measurements were carried out with the following settings: Intensity $=100 \mathrm{nW}$; Collection time $=5$ Seconds; Dead Time $=0.00545$ 
Seconds; Anode Voltage $=2,990 \mathrm{~V}$. The PYS data has been normalised to the same scale as the PESA data for ease of comparison.

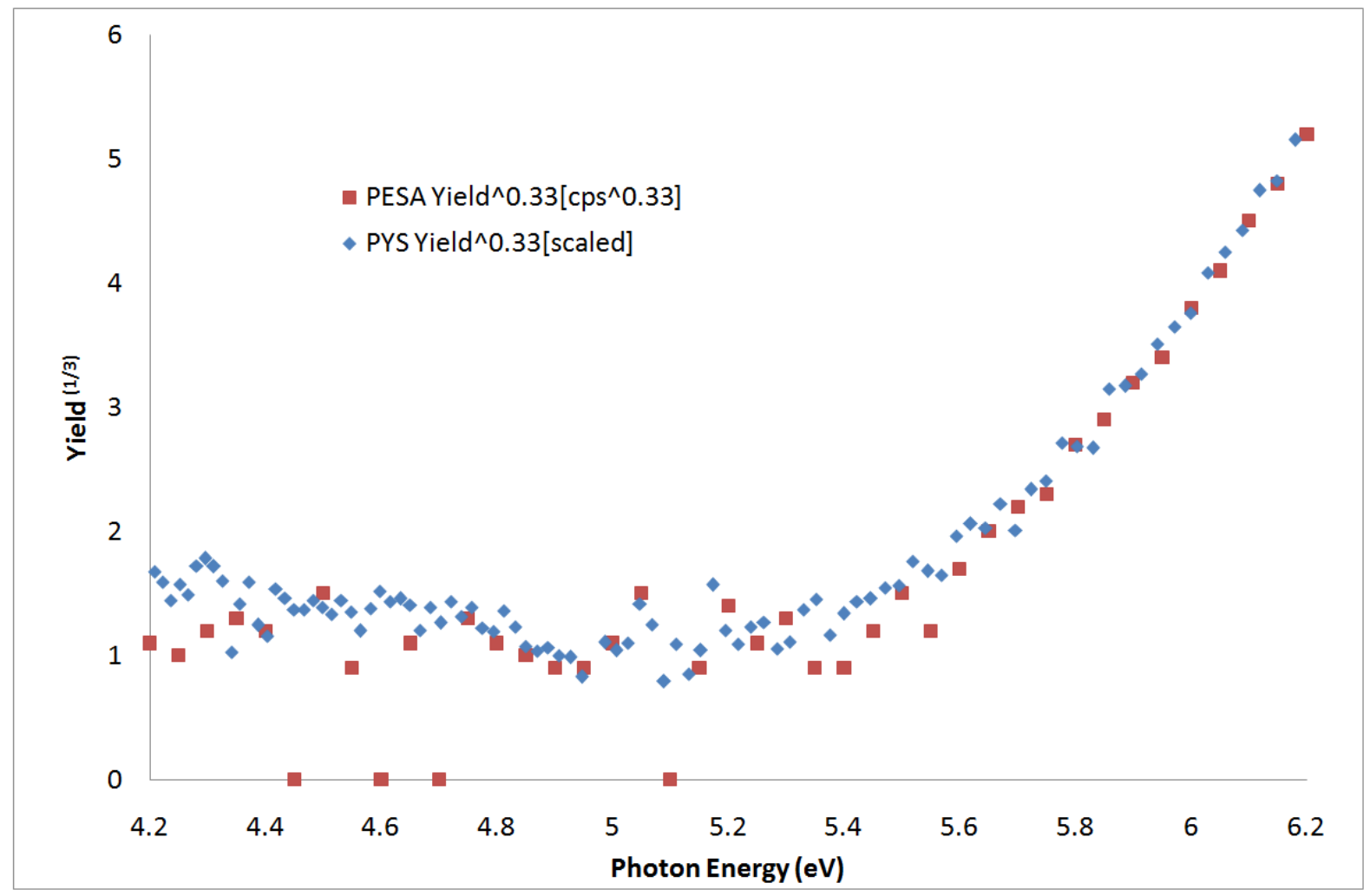

Figure 3: PYS PESA comparison - 5nm MoOx on a glass substrate

\subsection{Thin buffers on Multilayer Transparent Conducting Electrodes}

After confirmation of the effectiveness of our setup a series of tri-layer and 4-layer transparent electrodes were deposited, with the latter having high ionisation potential buffer layers and the trilayer being an anti-reflected very thin silver layer of the form $\mathrm{AZO} / \mathrm{Ag} / \mathrm{AZO}$ (AZO is aluminium doped zinc oxide) as described in figure 4. The silver layer is typically in the range 8 to $10 \mathrm{~nm}$ thick. In figure 4 to see the buffer impact on identical electrodes, one electrode is buffered, one is not, and both have been tested on flexible substrates and on glass. Buffering with thin $\mathrm{MoO}_{3-\mathrm{x}}$ has been reported on ITO (which is not flexible) [3] while $\mathrm{MoO}_{3-\mathrm{x}}$ has also been used not as a buffer but to sandwich and anti-reflect the silver layer in so called MAMs electrodes [11, 12]. The use of AZO as the silver AR layers for organic device flexible electrodes has not been previously reported. It yields better conductivity and transmittance than most other AR options. Conductivity improves in two ways from the AZO itself and because silver grows epitaxially on AZO which reduces scattering loss considerably in very thin Ag. The extrapolation of the PE plot to $4.6 \mathrm{eV}$ for un-buffered AZO in Fig. 4 yields its work function (not IP) as AZO is a transparent conducting oxide. (Undoped $\mathrm{ZnO}$ is an insulator and its PE spectra yield IP $=6.9$ to $7.7 \mathrm{eV}[13])$. This WF value of AZO is too low while buffering replaces WF with IP and provides the desired high range. Even though only $70 \mathrm{~nm}$ in total thickness these stacks have sheet resistance of just $10 \Omega / \mathrm{sq}$ and greater than $80 \%$ visible transmittance if optimized for air. The two AZO layers are the same thickness for air use designs but not if destined as an electrode for an organic device. For use in the device the optical design is changed to take account of the active organic layer adjacent to the electrode stack. The result is a thinner AZO layer facing the active side of the electrode. To increase the ionisation potential of this tri-layer thin film 
stack a $7 \mathrm{~nm}$ thin film buffer of a sub-stoichiometric MoOx is deposited on the device facing AZO layer with its supporting AZO thickness also adjusted for this layer in the device integrated multilayer design. The resulting shift in ionisation potential of the stack from $4.6 \mathrm{eV}$ to $5.4 \mathrm{eV}$ by the addition of a MoOx buffer layer is demonstrated in figure 4. Our IP values for $\mathrm{MoOx}$ in the range 5.3- $6.0 \mathrm{eV}$ are much lower than some for $\mathrm{MoO}_{3}$ of $9-10 \mathrm{eV}$ [14]. Our MoOx results include IPD emissions and in $\mathrm{MoO}_{3}$ just IPV is used for such high values. An important subtlety is also present in the MoOx data, namely that the main line in the PE spectra involves some overlap between IPV emission and IPD emission. Some insights into why this occurs, its manifestations in UPS, and why overlapping leads to the observed shifts in the main PE spectral line hence IP, can be found in two recent papers[15, 16]. One of these [16] also indicates that at a particular value of $x(\sim 2.6) \mathrm{MoOx}$ becomes metallic at which point and for lower $\mathrm{x}$ emission spectra will yield WF not IP. Our group will shortly publish results for complete Bulk Hetero Junction OPV devices, which use these highly flexible transparent conducting multilayer electrodes with optimized buffer layers on the anode.

\subsection{Other uses in device production}

We have also used this PYS tool in trouble-shooting for example to establish that care is needed in post deposition treatments and that some should be avoided. Indeed if possible it is preferable to build the final organic device on as-prepared fresh electrodes. For example when expected I-V characteristics and hence solar-to-electric conversion efficiencies have not been realised with electrodes that were known in their as-prepared form to have the desired high ionization potential the most common problem was that IP had dropped following cleaning with liquids and drying, or after discharge cleaning. Moderate heating of these thin oxides was also found to yield changes in IP. If electrode storage and/or transport is needed before building the final device on it, the storage atmosphere and any adjacent materials must be carefully chosen as they can also change IP. This compact, fast PYS tool allows easy checking if such impacts on IP are occurring.

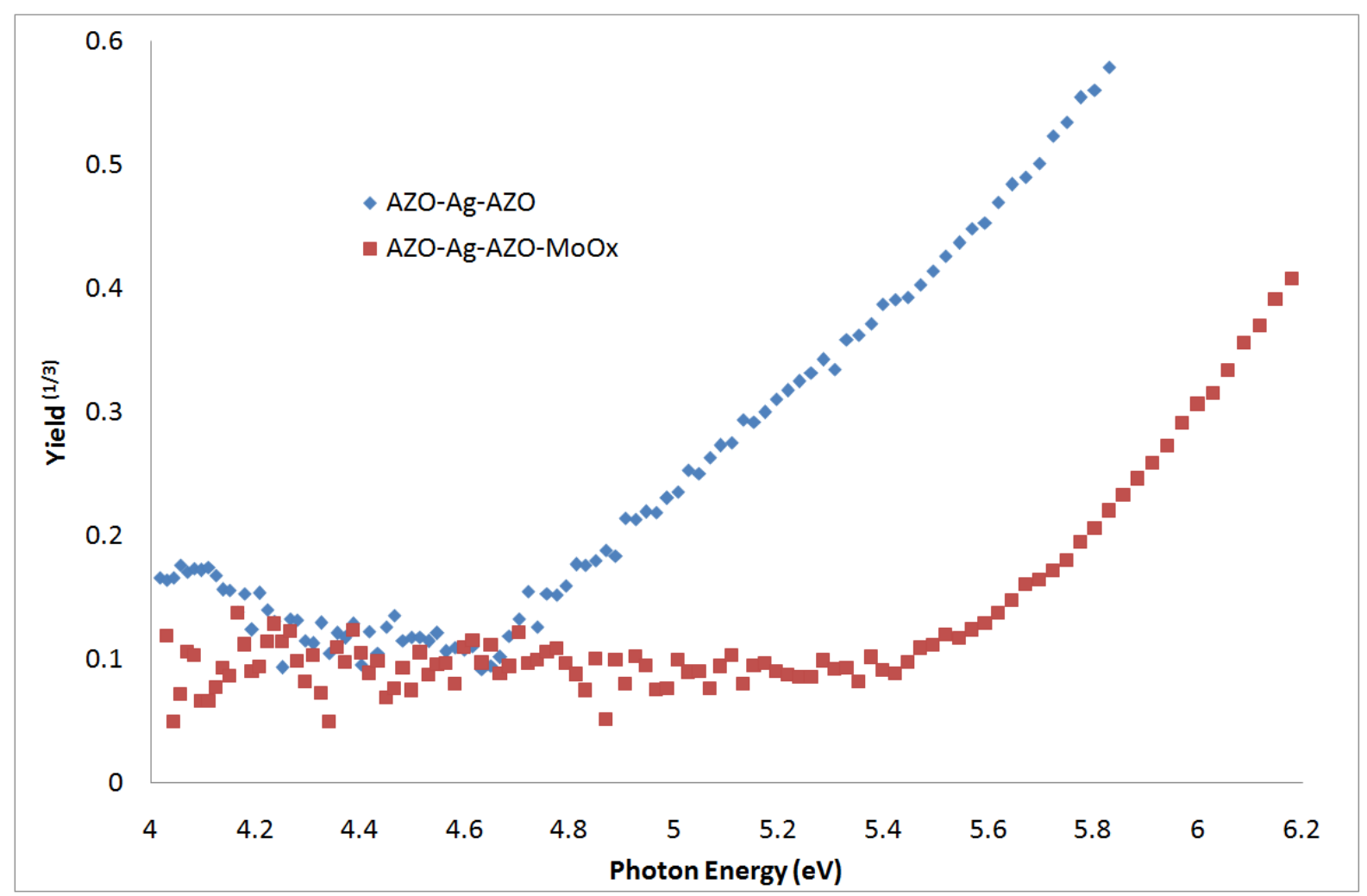

Figure 4: PYS Spectra, Multilayer Electrode with/without ionisation potential buffer layer 


\section{Conclusion}

We have demonstrated an effective method for easily assessing the photo-electron emission spectra from UV illuminated thin films. It has proved very useful in development of high ionization potential oxide buffer layers deposited on traditional and flexible multilayer transparent conducting electrodes to optimize charge transfer with the active organic layer in OPV. The simplicity of the method lends itself particularly well to assessment of variations in film deposition settings, and where applicable processing conditions. It has the ability to measure ionization potential accurately in hole transport buffer layer films such as NiOx and MoOx with thicknesses on the order of 5nm.

The method has been successfully applied, and where appropriate checked, for films and materials covering a wide range of conductivities from good insulator to good metal. No evidence of charging impacts on results, or of ion damage has been found.

\section{Acknowledgement}

This work was supported by the CSIRO Future Manufacturing Flagship: Flexible Transparent Electrodes for Plastic Electronics Cluster which includes The University of Queensland, University of Technology, Sydney and Flinders University. We would also like to thank Milos Toth and Toby Shanley of UTS for discussions regarding the PYS technique.

\section{References}

[1] G. Binning, H. Rohrer, C. Gerber, E. Weibel, Surface Studies by Scanning Tunneling Microscopy, Physical Review Letters, 49 (1982) 57-60.

[2] S. Tokito, K. Noda, Y. Taga, Metal oxides as a hole-injecting layer for an organic electroluminescent device, Journal of Applied Physics D, 29 (1996) 2750-2753.

[3] V. Shrotriya, G. Li, Y. Yao, C.-W. Chu, Y. Yang, Transition metal oxides as the buffer layer for polymer photovoltaic cells, Applied Physics Letters, 88 (2006) 073508-073508.

[4] M. Sebastiani, L.D. Gaspare, G. Capellini, C. Bittencourt, F. Evangelisti, Low-Energy Yield Spectroscopy as a Novel Technique for Determining Band Offsets: Application to the c-Si(100)/a-Si:H Heterostructure, Phys. Rev. Lett, 75 (1995) 3352-3355.

[5] M. Honda, K. Kanai, K. Komatsu, Y. Ouchi, H. Ishii, K. Seki, Atmospheric effect of air, N2, O2, and water vapor on the ionization energyof titanylphthalocyanine thin film studied by photoemission yield spectroscopy, Journal of Applied Physics, 102 (2007) 103704.

[6] L. Novak, M. Uncovsky, M. Toth, M. Cafourek, W. Parker, M. Straw, M. Emerson, Environmental Cell for Charged Particle Beam System, in, US Patent 2012/0112062 A1, 2012.

[7] A.L. Fletcher, B.L. Thiel, A.M. Donald, Amplification measurements of alternative imaging gases in environmental SEM., J. Phys. D: Appl. Phys. , 30 (1997) 2249-2257.

[8] J.M. Ballantyne, Effect of Phonon Energy Loss on Photoemissive Yield near Threshold, Phys. Rev. B, 6 (1972) 1436-1455.

[9] R.J. Davis, M.T. Lloyd, S.R. Ferreira, M.J. Bruzek, S.E. Watkins, M. Fahlman, J.E. Anthony, J.W.P. $\mathrm{Hsu}$, Determination of energy level alignment at interfaces of hybrid and organic solar cells under ambient environment, J. Mat. Chem., 21 (2011) 1721-1729.

[10] H. Kirihata, M. Uda, Externally quenched air counter for low\&\#x2010;energy electron emission measurements, Review of Scientific Instruments, 52 (1981) 68-70.

[11] H. Jin, C. Tao, M. Velusamy, M. Aljada, Y. Zhang, M. Hambsch, P. Burn, P. Meredith, Efficient, Large Area ITO-and-PEDOT-free Organic Solar Cell Sub-modules, Advanced Materials, 24 (2012) 2572-2577. 
[12] X.-Y. Jiang, Z.-L. Zhang, J. Cao, M.A. Khan, Khizar-ul-Haq, W.-Q. Zhu, White OLED with high stability and low driving voltage based on a novel buffer layer MoOx, , Journal of Physics D: Applied Physics 40 (2007) 5553-5557.

[13] A. Klein, C. Körber, A. Wachau, F. Säuberlich, Y. Gassenbauer, R. Schafranek, S.P. Harvey, T.O. Mason, Surface potentials of magnetron sputtered transparent conducting oxides, Thin Solid Films, 518 (2009) 1197-1203.

[14] M. Kröger, S. Hamwi, J. Meyer, T. Riedl, W. Kowalsky, A. Kahn, Role of the deep-lying electronic states of $\mathrm{MoO} 3$ in the enhancement of hole-injection in organic thin films,, Appl. Phys. Lett., 95 (2009) 123301.

[15] M. Vasilopoulou, A.M. Douvas, D.G. Georgiadou, L.C. Palilis, S. Kennou, L. Sygellou, I. Kostis, A. Soultati, G. Papadimitropoulos, D. Davazoglou, P. Argitis, The Influence of Hydrogenation and Oxygen Vacancies on Molybdenum Oxides Work Function and Gap States for Application in Organic Optoelectronics,, J. Am. Chem. Soc., 134 (2012) 16178.

[16] G.B. Smith, D. Golestan, A.R. Gentle, The insulator to correlated metal phase transition in molybdenum oxides, Appl. Phys. Lett., In Press (2013). 